\title{
CONTRIBUIÇÃO DA CULTURA ORGANIZACIONAL PARA A GESTÃO DO CONHECIMENTO NO CONTEXTO DA GESTÃO DA CADEIA DE SUPRIMENTOS
}

\section{CONTRIBUTION OF ORGANIZATIONAL CULTURE TO KNOWLEDGE MANAGEMENT IN THE CONTEXT OF SUPPLY CHAIN MANAGEMENT}

Ana Livia Cazanea

Marta Lígia Pomim Valentimb

\begin{abstract}
RESUMO
Objetivo: Buscou-se mais bem compreender o objeto pesquisado, a partir da abordagem da gestão da cadeia de suprimentos, debatendo de modo inter-relacionado as contribuições da cultura organizacional e da gestão do conhecimento. Metodologia: Trata-se de uma pesquisa de abordagem qualitativa, do tipo bibliográfico. Resultados: Constatou-se que a relação entre cultura organizacional, gestão do conhecimento e gestão da cadeia de suprimentos é real e direta, uma vez que a cultura contribui para a efetividade dos processos de ambos os modelos de gestão. Destaca-se que a forte relação se deve ao fato de que a cultura organizacional é determinante para o estímulo ou desencorajamento do compartilhamento de conhecimento. Em tese, o estímulo à adoção de práticas de compartilhamento de conhecimento pode proporcionar diferenciais competitivos para a cadeia, entretanto, na prática o compartilhamento é uma ação difícil de ser realizada, devido aos valores individuais e organizacionais que influem, tanto no contexto interno da organização quanto no coletivo da cadeia de suprimentos. Conclusões: A partir desta constatação, sugere-se que as organizações devam desenvolver uma cultura voltada à valorização da informação enquanto recurso estratégico, enfocando o melhor uso da gestão do conhecimento, apoiando as atividades de compartilhamento. Para a melhoria do desempenho organizacional é essencial cultivar uma cultura que incentive e forneça oportunidades para comunicar ideias, conhecimentos e experiências.
\end{abstract}

Descritores: Cultura Organizacional. Gestão do Conhecimento. Gestão da Cadeia de Suprimentos. Compartilhamento de Conhecimento.

\footnotetext{
a Doutoranda em Ciência da Informação pela Universidade Estadual Paulista Julio de Mesquita Filho (UNESP). E-mail: al.cazane@unesp.br

b Doutora em Ciências da Comunicação pela Universidade de São Paulo (USP). Docente do Programa de Pós-Graduação em Ciência da Informação da Universidade Estadual Paulista (PPGCI-UNESP). E-mail: valentim@valentim.pro.br.
} 


\section{INTRODUÇÃO}

Considera-se a Cultura Organizacional (CO) um elemento totalmente imbricado à dinâmica organizacional, visto que é essencial para o desenvolvimento de qualquer processo realizado em seu ambiente. A cultura organizacional abrange princípios, valores, crenças, ritos, mitos, entre outros aspectos que regem o comportamento dos sujeitos organizacionais. Nessa perspectiva, a cultura organizacional pode ser estimuladora ou desestimuladora de comportamentos positivos relacionados ao compartilhamento e socialização de conhecimento no contexto organizacional.

As organizações contemporâneas necessitam gerenciar o conhecimento produzido em seu ambiente, de modo a criar diferenciais competitivos para o seu desenvolvimento e atuação no mercado internacionalizado. Nesse intuito, a Gestão do Conhecimento (GC) é um modelo de gestão que pode contribuir para valorizar e reter o capital intelectual gerado na organização.

A GC, compreendida como um modelo de gestão, viabiliza ou inviabiliza a melhoria de processos que, por sua vez, impactam na qualidade e produtividade da organização. Os conhecimentos e aprendizagens dos sujeitos organizacionais são fundamentais para a evolução do negócio organizacional.

A abordagem deste texto se volta para a Gestão da Cadeia de Suprimentos (GCS) [Supply Chain Management (SCM), em inglês] que abrange pessoas, processos, informações e recursos na atividade de transportar produtos e/ou serviços dos fornecedores aos clientes, ou seja, abrange toda a operação da organização, visando aprimorar a eficiência de seus processos e obter resultados eficazes no curto, médio e longo prazos. A GCS é responsável pelos processos e fluxos da organização, bem como pela inter-relação com seus stakeholders.

O Council of Supply Chain Management Professionals (CSCMP) explica que a GCS planeja e gerencia todas as atividades que envolvem a logística organizacional, desde a aquisição (fornecedores), venda (funcionários), até a entrega (intermediários e clientes) de produtos e serviços produzidos por uma determinada organização. Dessa maneira, a GCS envolve atores internos e 
externos que, por sua vez, influem significativamente no negócio organizacional (CSCMP, 2005).

Nesse sentido, buscou-se mais bem compreender o objeto pesquisado, a partir da abordagem da GCS, debatendo de modo inter-relacionado as contribuições da CO e da GC. Parte-se do pressuposto de que implantar a GC em uma organização requer primeiramente uma mudança cultural, cujas barreiras organizacionais precisam ser diminuídas ou eliminadas para que se possa atingir toda a cadeia do segmento econômico envolvido.

Este trabalho contempla, além da introdução, a seguinte estrutura: na Seção 2 são descritos os procedimentos metodológicos, na sequência, o referencial teórico aborda a GC, a GCS, finalizando o conteúdo com a apresentação da CO como fator determinante para a GC no contexto da GCS. $\mathrm{Na}$ última Seção são apresentadas as considerações finais deste estudo.

\section{METODOLOGIA}

Esta pesquisa de abordagem qualitativa, do tipo bibliográfico, enfocou os temas GC, GCS e CO nos campos da Ciência da Informação e Administração. Marconi e Lakatos (2006, p. 71) mencionam que a revisão "[...] bibliográfica não é mera repetição do que foi dito ou escrito sobre certo assunto, mas propicia o exame de um tema sob novo enfoque ou abordagem, chegando a conclusões inovadoras". Gil (2008, p. 44) explica que a revisão bibliográfica é:

[...] desenvolvida com base em material já elaborado, constituído principalmente de livros e artigos científicos. Embora em quase todos os estudos seja exigido algum tipo de trabalho dessa natureza, há pesquisas desenvolvidas exclusivamente a partir de fontes bibliográficas.

A revisão de literatura definiu como fontes de informação a serem pesquisadas: Web of Science (WoS); Portal de Periódicos da Coordenação de Aperfeiçoamento de Pessoal de Nível Superior (CAPES); Scientific Electronic Library Online (SciELO); Base de Dados Referenciais de Artigos de Periódicos em Ciência da Informação (BRAPCI), selecionadas pela confiabilidade dos resultados e facilidade de busca. A pesquisa compreende o período de 2000 a 2020, além dos textos clássicos das áreas de Ciência da Informação e 
Administração anteriores ao referido período.

Após o levantamento sobre os temas supracitados, os textos foram fichados e analisados. A inclusão dos textos foi determinada pela relevância e aderência à temática discutida neste artigo. Assim, os critérios de inclusão foram:

- Artigos que abordassem os temas GC, GCS e CO, bem como a interdisciplinaridade entre eles;

- Trabalhos publicados e disponíveis integralmente;

- Textos pulicados em português ou inglês.

A partir da análise dos textos pré-selecionados, foram excluídos os estudos que não possuíam versões completas disponíveis gratuitamente ou pelos acessos fornecidos pela Universidade Estadual Paulista (Unesp); textos apresentados no formato de pôster, palestras, workshops, resumos, resenhas; trabalhos de conclusão de cursos (graduação); textos em outros idiomas que não os supracitados e documentos que após a leitura completa não demostraram aderência com a temática da pesquisa.

Dessa maneira, foi possível reunir elementos para promover uma reflexão consistente visando inter-relacionar os conceitos convergentes e divergentes encontrados na literatura. A partir das reflexões e inferências foi possível perceber a importância da cultura organizacional para GC no contexto da GCS.

\section{GESTÃO DO CONHECIMENTO}

A GC é um tema que desperta interesse tanto nas organizações quanto na academia, no contexto global atual as organizações bem-sucedidas são as que criam novos conhecimentos consistentes, isso ocorre porque o mercado está em constante transformação, a tecnologia em avanço rápido, e a competitividade crescente. Esse cenário de significativa competitividade requer inovação constante que, por sua vez, é gerada por pessoas, ou seja, são os sujeitos organizacionais que criam e disseminam novos conhecimentos. Nesse intuito, torna-se necessário realizar a GC de maneira plena, uma vez que o conhecimento é compreendido como um ativo intangível ou imaterial de valor estratégico para as organizações.

Em vista disso, dada a compreensão desta importância para o 
crescimento organizacional, é relevante buscar na literatura textos que abordem a GC e, para tanto, é necessário evidenciar a informação enquanto propulsora do conhecimento.

De acordo com Choo (2003), a informação é um importante recurso intrínseco de todas as atividades e processos organizacionais. Vale destacar que a informação nasce a partir da percepção e compreensão de um conjunto de dados pelo indivíduo, e somente após a apropriação e o estabelecimento de inter-relações pelo sujeito organizacional é que pode se transformar em 'novo' conhecimento ou conhecimento incremental e, este por sua vez, pode ser aplicado a partir de uma ação (como, por exemplo, uma tomada de decisão).

As organizações necessitam ser capazes de perceber a importância de suas fontes de informação e, para tanto, precisam usar de modo pleno as Tecnologias de Informação e Comunicação (TIC) em seus processos organizacionais.

No contexto organizacional, a informação pode se constituir em objeto ou fenômeno, sendo analisada e utilizada individualmente ou coletivamente, como um elemento único ou como parte de um processo (VALENTIM, 2008). Ao compreender a informação enquanto recurso, torna-se necessário realizar delimitações entre outros dois componentes que se relacionam, interagem e sofrem transformações na relação com a informação, o dado e o conhecimento.

De acordo com Davenport e Prusak (1998), pode-se definir 'dado' como simples observações sobre o estado do mundo, ou seja, são dados registrados com o auxílio de algum suporte; na maioria das vezes, com o auxílio de tecnologias. A 'informação' é definida como um conjunto de dados dotados de relevância e propósito, ou seja, dados percebidos, compreendidos e contextualizados por um sujeito cognoscente. $O$ 'conhecimento' é definido como algo que reside na mente humana, construído na relação do indivíduo com o mundo.

Nesse sentido, Ponjuán Dante (2004) destaca que a informação depende de dados que, podem se transformar em informação ao receberem significado atribuído por um indivíduo, a partir de distintos processos de agregação de valor e de um determinado contexto. Validando essa ideia, Almeida Júnior (2008) 
conceitua 'informação' como algo subjetivo, intangível e dependente do indivíduo.

A informação se caracteriza como intermediadora no processo de construção do conhecimento. A informação é um componente que nasce da intermediação entre o sujeito e os dados percebidos, sendo utilizada temporariamente no processo de construção de conhecimento. A informação pode vir e existir a partir do contato do indivíduo com o suporte (digital e/ou analógico) que contém o conjunto de dados que, uma vez compreendidos, podem ser apropriados pelo indivíduo e transformarem-se em uma informação. Como premissa, entende-se a informação a partir da modificação, da mudança, da reorganização, da reestruturação, enfim, da transformação do conhecimento (ALMEIDA JÚNIOR, 2008).

Para Valentim (2002), a informação é compreendida como matéria-prima, ou seja, insumo básico de fluxos e processos sociais, a comunicação/telecomunicação como meio de disseminação e as TIC como infraestrutura de armazenagem, processamento e acesso. Nesse contexto, destaca-se a importância do sujeito no processo de transformação dos dados em informação e da informação em conhecimento.

Evidencia-se que a GC está relacionada à Gestão da Informação (Gl), assim como vale destacar que há dependência entre ambas, pois se 0 conhecimento gerado não é explicitado em suportes informacionais, não há informação para ser gerenciada. Da mesma maneira, se não há informação organizada, analisada, armazenada, acessível, não é possível transformá-la em insumo para a criação de conhecimento. Se por um lado a Gl centra seus esforços no negócio da organização, a gestão do conhecimento centra seus esforços no capital intelectual existente nesse ambiente (VALENTIM; JORGE; CERETTA-SORIA, 2014).

A complexidade relacionada à gestão do conhecimento reside no tratamento dado a dois componentes básicos: conhecimento tácito e explícito. De acordo com Nonaka e Takeuchi (1997), a estrutura conceitual básica do conhecimento tem base em duas dimensões - epistemológica e ontológica. $\mathrm{Na}$ dimensão ontológica, em termos estritos, o conhecimento só é criado por 
indivíduos. Uma organização não pode gerar conhecimento sem indivíduos e, estes, quando criativos, devem ser apoiados ou thes deve ser proporcionado contextos para a criação do conhecimento.

A dimensão epistemológica baseia-se na distinção entre conhecimento tácito e explícito. Para Takeuchi e Nonaka (2008) o conhecimento é formado por esses dois componentes que aparentemente são opostos, entretanto são interrelacionados. Os sujeitos organizacionais geram novos conhecimentos, por meio da transformação do conhecimento tácito em conhecimento explícito e viceversa, assim, cabe a organização propiciar as condições necessárias para gerenciar tanto um quanto o outro, ou seja, o conhecimento explícito por meio da Gl e o conhecimento tácito por meio da GC.

Pode-se definir conhecimento explícito como sendo o conhecimento que é expresso por meio de signos e símbolos (palavras, números, sons, imagens etc.), que pode ser compartilhado no formato de dados, fórmulas, recursos visuais, especificações de produtos ou manuais. Assim, o conhecimento explícito pode ser rapidamente transmitido aos indivíduos (TAKEUCHI; NONAKA, 2008).

Por outro lado, o conhecimento tácito não é facilmente visível e explicável, tornando sua gestão mais difícil, uma vez que se trata de um conhecimento pessoal, dificultando sua formalização e tornando as atividades de comunicação e compartilhamento mais complexas. O conhecimento tácito pode ser gerado em reuniões, eventos, construção individual de conhecimento, valores, crenças e comportamento organizacional, experiências práticas, aprendizagem ao longo da vida e/ou conhecimento de mundo. Destaca-se que o conhecimento tácito é percebido em ações e práticas exercidas pelo indivíduo, refletindo os ideais, valores ou emoções que o sujeito incorpora (WIIG, 1993; VALENTIM, 2004; TAKEUCHI; NONAKA, 2008).

Valentim (2008, documento eletrônico não paginado) explica que a GC é um modelo de gestão integrada voltada aos fluxos informais da organização e, assim, desenvolve ações direcionadas ao compartilhamento e socialização do conhecimento. A autora destaca dez atividades básicas da GC: a) Identificar necessidades/demandas de conhecimento; b) Mapear e reconhecer fluxos informais (redes); c) Desenvolver a cultura organizacional positiva em relação ao 
compartilhamento/socialização de conhecimento; d) Proporcionar a comunicação informacional de forma eficiente, utilizando tecnologias de informação e comunicação; e) Criar espaços criativos dentro da organização; f) Desenvolver competências e habilidades voltadas ao negócio da organização; g) Criar mecanismos de captação e sistematização de conhecimento, gerado por diferentes pessoas da organização; h) Desenvolver e implantar sistemas de diferentes naturezas, visando o compartilhamento e uso de conhecimento; i) Elaborar e implantar normatizações visando à sistematização do conhecimento gerado internamente; j) Retroalimentar o ciclo (Figura 1).

\section{Figura 1 - Modelo de Gestão do Conhecimento}

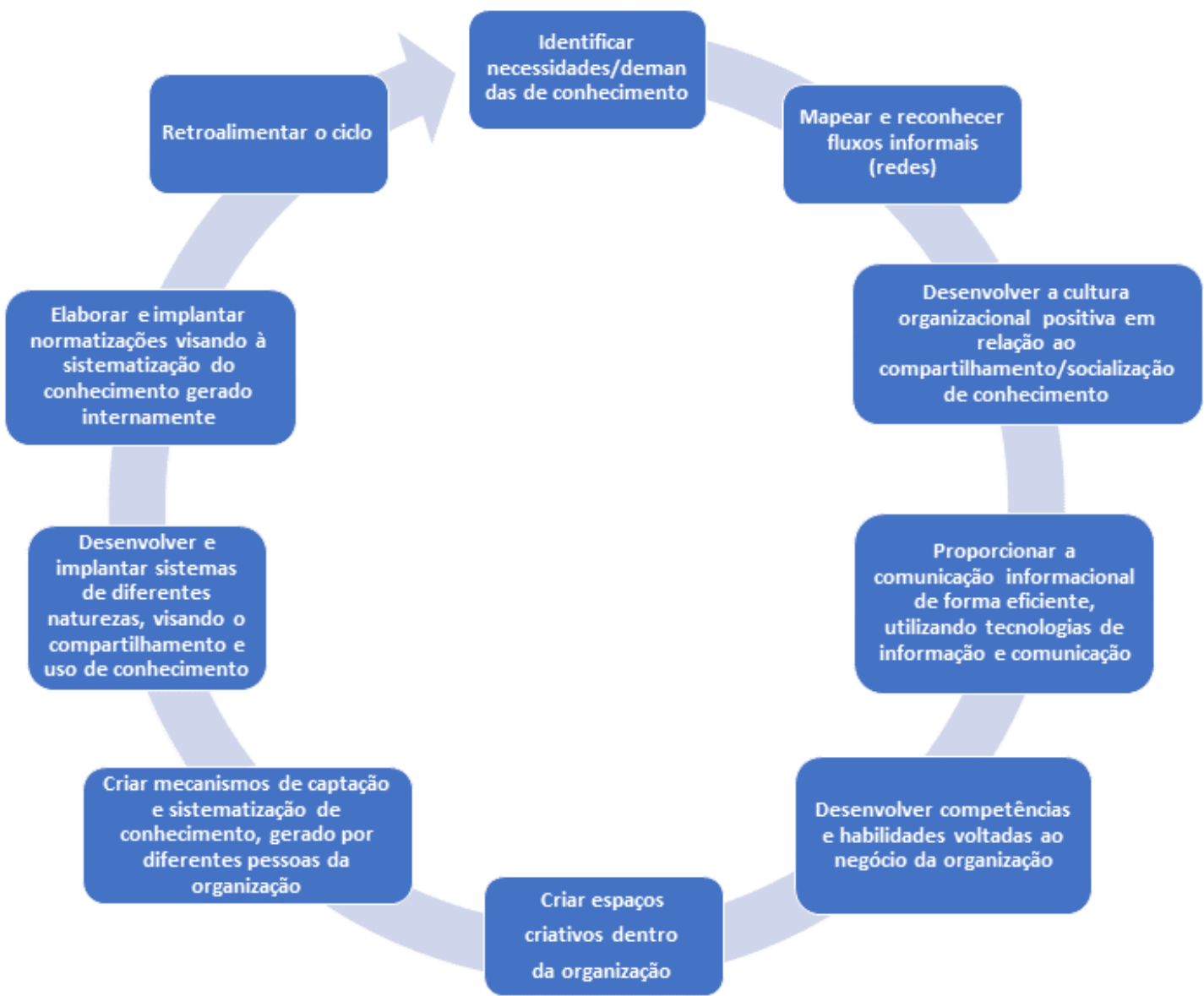

Fonte: Elaborada a partir do texto de Valentim (2008)

Enquanto recurso organizacional, a GC ocorre no momento em que as organizações de diferentes segmentos econômicos passam a perceber a importância do conhecimento como um ativo essencial para seu 
desenvolvimento, "[...] como uma nova mercadoria e uma moeda potencial para explicar a nova teoria econômica e estimular os motores econômicos de muitas nações" (SUTTON, 2007, p. 287).

Nessa perspectiva, organizações de diferentes segmentos passaram a gerenciar o conhecimento enquanto ativo organizacional capaz de proporcionar diferenciais competitivos que, quando bem gerenciado, pode proporcionar ganhos significativos para todos os agentes envolvidos.

\section{GESTÃO DA CADEIA DE SUPRIMENTOS}

De acordo com Lambert, Cooper e Pagh (1998), a expressão 'Gestão Cadeia de Suprimentos' foi originalmente introduzida por consultores empresariais no começo dos Anos 1980, passando a atrair a atenção dos profissionais e acadêmicos atuantes nessa área. Outros autores argumentam que a GCS vem sendo praticada há décadas, porém, com nomes diferentes e com diferentes níveis de integração.

Para Mentzer et al. (2001) uma cadeia de suprimentos é definida como um conjunto de três ou mais entidades (organizações ou indivíduos) envolvidos diretamente nos fluxos a montante e a jusante de produtos, serviços, finanças e/ou informações de um ponto de origem para um cliente. A visão sistêmica possibilita que as organizações compreendam os efeitos de suas ações em toda a cadeia da qual faz parte, proporcionando uma visão real sobre sua posição (TAN, 2001).

A GCS trata basicamente da integração holística dos processos de negócios (business processes) por intermédio da cadeia produtiva, com objetivo de atender o consumidor final efetivamente, isto é, sendo eficiente e eficaz simultaneamente (PIRES et al., 2001). De modo geral, a GCS busca intensificar, somar e amplificar os benefícios de uma gestão integrada da cadeia de suprimentos. Assim, as estratégias e as decisões deixam de ser formuladas e firmadas sob a perspectiva de uma única organização e passam a fazer parte da cadeia produtiva como um todo (PARRA; PIRES, 2003).

Além disso, Mentzer et al. (2001) definem a GCS como a coordenação sistêmica das funções organizacionais estratégicas, táticas e operacionais, seja 
de uma organização específica, seja de todos os negócios que fazem parte da cadeia de suprimentos, visando a melhoria do desempenho das organizações individualmente, bem como da cadeia de suprimentos como um todo no longo prazo.

Outra definição - desenvolvida em 1994 pelos membros do Global Supply Chain Forum (GSCF) e, posteriormente, modificada em 1998 -, da GCS se refere a integração dos processos de negócios desde o usuário final até os fornecedores originais (primários), responsáveis por providenciar produtos, serviços e informações capazes de adicionar valor para clientes e stakeholders (PIRES, 2009).

Essas definições são convergentes, uma vez que evidenciam a importância da integração dos agentes envolvidos durante todo o processo. Assim, os temas 'estratégia' e 'diferencial competitivo' podem ser discutidos, visto que o relacionamento nas cadeias de suprimento é um item estratégico para o sucesso das organizações (BANDEIRA; MELLO; MAÇADA, 2009). Nesse processo, em que empresas têm a possibilidade de trabalhar com fornecedores, clientes e mercados de maneira integrada, a necessidade de coordenação e colaboração entre os diversos agentes envolvidos é fundamental (VIVALDINI; PIRES, 2010).

Entretanto, as organizações precisam estar dispostas a confiar e comprometer-se com seus fornecedores e clientes, tornando-se interdependentes, com a visão e os processos-chave voltados para toda a cadeia de suprimentos (LI et al., 2005). Segundo Oliveira e Cohen (2010), nos últimos anos as metodologias aplicadas à GCS avançaram para uma tendência colaborativa, na qual as organizações passaram a aproximar-se dos parceiros como estratégia de melhoria de seus processos.

A gestão colaborativa na cadeia de suprimentos passou a ser mais discutida na literatura a partir do avanço tecnológico surgido em meados de 1990, e foi intensificada na primeira década do Século XXI. No entanto, Vivaldini e Pires (2010) ressaltam que juntamente com a evolução do conceito, percebese a dificuldade de sua aplicação. Machline (2011), por exemplo, destaca o fato de as organizações nacionais, ainda, encontrarem-se no limiar desse paradigma 
de colaboração, pelo receio de revelar dados confidenciais aos fornecedores, que poderiam divulgá-los aos concorrentes.

Segundo Vivaldini e Pires (2010), isso tende a ocorrer porque a cultura desenvolvida na cadeia geralmente não privilegia ações colaborativas, nem valoriza a importância do desenvolvimento do negócio para todos os agentes, o que torna a visão de cada organização limitada e individualizada. Nesse contexto, o desafio é encontrar meios para facilitar ações colaborativas na cadeia de suprimentos.

Considerando este cenário, destaca-se a importância da confiança, cujo conceito na literatura é amplamente associado ao conceito de cooperação (BANDEIRA; MELLO; MAÇADA, 2009). Não existe uma definição única para o conceito de confiança. Alguns autores a compreende como sendo a expectativa sobre o modo de atuação desejado do confiado, enquanto outros a definem em termos da avaliação sobre a boa vontade e a confiabilidade do outro em uma situação de risco (NIELSEN, 2004). Para Ganesan (1994), a confiança pode ser definida como uma conviç̧ão, sentimento ou expectativa sobre um parceiro, resultante de sua confiabilidade e intenções, e possui dois componentes principais. O primeiro é a credibilidade, relacionada à percepção de que 0 parceiro tem experiência necessária para realizar sua atividade de maneira efetiva e confiável. O segundo é a benevolência, crença em que o parceiro continuará agindo de modo apropriado ao relacionamento, mesmo em situações adversas, não tomando nenhuma atitude que possa afetar a organização (GANESAN, 1994; MIN; MENTZER, 2004).

A confiança também diminui a percepção de risco de comportamento oportunístico entre organizações, amplia a convicção de que os conflitos serão resolvidos de maneira justa e age como uma salvaguarda informal, que encoraja relacionamentos de longo prazo, compartilhamento de informação e conhecimento. Também se constitui em um fator relevante para a redução de custos de transação, ao evitar necessidade de revisão e manutenção de contratos (GANESAN, 1994; DYER; SINGH, 1998).

Empiricamente, Kaufmann e Carter (2006) verificaram que a confiança promove a cooperação e o compartilhamento de informações, por reduzir a 
necessidade de monitoramento de contratos, especificações e incertezas, ao mesmo tempo em que promove investimentos no relacionamento e em ativos específicos.

Uma das principais vantagens da cooperação na cadeia de suprimentos é o maior compartilhamento de informações. Entretanto, em alguns casos, os membros que detêm mais informações e possuem uma boa estrutura de TIC exercem maior controle sobre a cadeia (BANDEIRA; MELLO; MAÇADA, 2009). Considerando os aspectos apresentados e discutidos até o momento, percebese que a evolução histórica dos conceitos caminha para a geração de vantagem competitiva, por meio de um maior compartilhamento de informações e cooperação.

Há um crescente reconhecimento de que a GCS oferece às organizações oportunidades significativas para desenvolver a vantagem estratégica em relação aos concorrentes (WEN; GU, 2014). As organizações reconhecem que não podem mais competir efetivamente isolando-se de seus fornecedores ou de outras entidades na cadeia de fornecimento (LUMMUS; VOKURKA, 1999). Assim, a temática voltada à GCS tem forçado constantemente pesquisadores e gestores a pensar as operações e processos, por meio das fronteiras organizacionais (MELNYK et al., 2007).

Nessa perspectiva, as organizações podem obter vários benefícios para suas cadeias de suprimentos a partir da colaboração e compartilhamento de informações, incluindo a geração de vantagem competitiva por meio do gerenciamento inteligente de estoques, desenvolvimento de novos produtos e de novos conhecimentos.

\section{CULTURA ORGANIZACIONAL COMO FATOR DETERMINANTE PARA A GESTÃO DO CONHECIMENTO NO CONTEXTO DA GESTÃO DA CADEIA DE SUPRIMENTOS}

A cultura organizacional é extremamente importante para qualquer tipo de organização, os estudos sobre a cultura organizacional têm se desenvolvido ao ponto de se tornarem cada vez mais relevantes para empresários, consultores e pesquisadores da área de gestão, uma vez que ela influi diretamente nas 
estratégias, objetivos e metas organizacionais.

Nesse contexto, as organizações podem ser consideradas como sociedades humanas, afinal, todas as organizações dependem de pessoas. Desse modo, abordar a cultura organizacional é abordar uma determinada sociedade sob um viés complexo, que apresenta inúmeros aspectos além daqueles que aparecem na estrutura formal das organizações.

Dessa maneira, percebe-se 0 crescimento dos estudos sobre 0 comportamento humano nas organizações a partir da influência da Escola das Relações Humanas. Além disso, pode-se destacar a crescente necessidade de entender o homem e seu comportamento no ambiente organizacional, em função das mudanças constantes nas estratégias de mercado, vendas, produção e nas organizações como um todo em função da revolução científico-tecnológica que vivenciamos (DIAS, 2013).

Schein (1995, p. 8-9) apresenta uma definição de cultura organizacional, a saber:

Cultura é a experiência que o grupo adquiriu à medida que resolveu seus problemas de adaptação externa e integração interna, e que funciona suficientemente bem para ser considerada válida. Portanto, essa experiência pode ser ensinada aos novos integrantes como forma correta de perceber, pensar e sentir-se em relação a esses problemas.

Ainda, segundo o autor supracitado, se realmente se quer interpretar a cultura de uma organização, deve-se ir além da superfície da organização - além dos artefatos visíveis e descobrir os pressupostos básicos fundamentais -, isto é, como as relações que são o centro da cultura de uma organização ocorrem. Para se descrever a cultura de uma organização é preciso recuperar sua missão, visão, objetivos e metas, identificar os principais períodos de formação da cultura que, por sua vez, são marcados por aspectos invisíveis e visíveis como, por exemplo, o layout da organização, o vestuário, os valores (as pessoas reagem a determinadas situações com comportamentos padrão, repetitivos), e por pressupostos básicos, que normalmente são inconscientes, mas que na realidade determinam como os membros do grupo percebem, pensam e sentem (FLEURY; SHINYASHIKI; STEVANATO, 1997).

De acordo com Robbins (2007) a cultura desempenha várias funções, 
entre elas destaca: define fronteiras; propicia o senso de identidade; facilita 0 comprometimento de seus membros; estimula a estabilidade em relação à dinâmica organizacional; influi no comportamento de seus membros.

Complementando este pensamento, Ritter (2008) explica que a cultura organizacional possui quatro funções básicas no contexto organizacional: 1) Identificação: propicia aos seus membros conhecer/reconhecer a identidade organizacional; 2) Integração: proporciona um elo entre seus membros de modo a conhecer/reconhecer o que os une; 3) Coordenação: possibilita por meio da aplicação de normas uma maior liberdade de ação e decisão; 4) Motivação: influi e legitima o comportamento de seus membros de modo a buscar o bem comum para atingir os objetivos e metas planejados pela organização.

Assim, a cultura de uma organização é um sistema de valores e crenças compartilhados que influenciam o comportamento daqueles que a compartilham.

Considerando este cenário organizacional, percebe-se a importância e a complexidade que envolvem a cultura de uma organização, influenciando o ambiente organizacional em vários níveis e impactando diretamente em fatores como inovação, criatividade e competitividade. Assim, de acordo com Tseng (2010), o papel da cultura organizacional está fortemente associado ao desempenho competitivo da organização.

Nessa perspectiva, a cultura organizacional influencia tanto a estrutura quanto os processos organizacionais que, por sua vez, influenciam nas estratégias desenvolvidas para alcançar os objetivos e metas definidos no contexto da organização. Exatamente por isso ela é determinante para qualquer tipo de modelo de gestão.

Neste artigo pretende-se expandir o conceito, a partir da abordagem da GCS. Se atualmente a competitividade ocorre entre cadeias e essas cadeias são formadas por organizações que desenvolvem diferenciais competitivos por meio da GC, qual é o impacto da cultura organizacional nesse contexto?

A cultura pode ajudar a definir os valores fundamentais e os princípios que norteiam o comportamento organizacional, bem como entre as organizações da mesma cadeia de suprimentos. Nesse contexto, a cultura organizacional é tão importante quanto as ferramentas e meios tecnológicos de gerenciamento de 
informações. O humano está no centro de sua implementação (ABDESSADAK; ACHELHI; REKLAOUI, 2018).

De acordo com Al-Tit (2017), a GC só pode ser concebida em uma abordagem de confiança em um relacionamento ganha-ganha, criando uma cultura organizacional equilibrada e sustentável. Por outro lado, uma cultura mal adaptada está na raiz da maioria das falhas ao tentar se implantar a GC.

Sendo assim, torna-se indispensável o papel da cultura organizacional, pois a cultura de compartilhamento e confiança é um fator crítico de sucesso da GC (AL-TIT, 2017; ABDESSADAK; ACHELHI; REKLAOUI, 2018), visto que nenhuma tecnologia por si só pode cuidar das pessoas, frequentemente ignoradas na parte cultural, impactando na intensidade e na qualidade da comunicação entre os sujeitos organizacionais.

Considerando o cenário da GC no contexto da GCS, destaca-se a importância da confiança entre as organizações de uma mesma cadeia de suprimentos. De acordo com Bandeira, Mello e Maçada (2009), a confiança é um conceito que está presente na literatura e é amplamente associado ao conceito de cooperação.

Ressalta-se que a GCS depende da GC e das organizações que interagem ao longo do processo produtivo. Dessa maneira, evidencia-se que a cultura organizacional pode estimular a interação e a confiança ou reprimir essas trocas/compartilhamentos, ou seja, a cultura organizacional pode potencializar a GC na cadeia de suprimentos ou impedir que ela aconteça de maneira efetiva.

Pode-se destacar, também, a questão do 'poder', em uma cadeia são diversas as formas por meio das quais as organizações mais fortes podem exercer dominação sobre os demais membros da cadeia. Munson et al. (1999) alegam que as organizações podem exercer o controle através de cinco áreas: 1. controle de preços; 2 . controle da estrutura do canal; 3 . controle de operação; 4. controle da informação; 5 . controle de inventário.

As organizações dominantes não apenas possuem uma cultura organizacional que reforça esses aspectos, como também influenciam a cultura de todas as demais organizações envolvidas na cadeia de suprimentos.

Nesse contexto, todos os processos e práticas da GCS dependem 
fundamentalmente da cooperação e confiança entre os agentes da cadeia que, por sua vez, precisam compartilhar dados, informações e conhecimento, para juntos construírem vantagem competitiva.

Figura 2 - Dinâmica organizacional

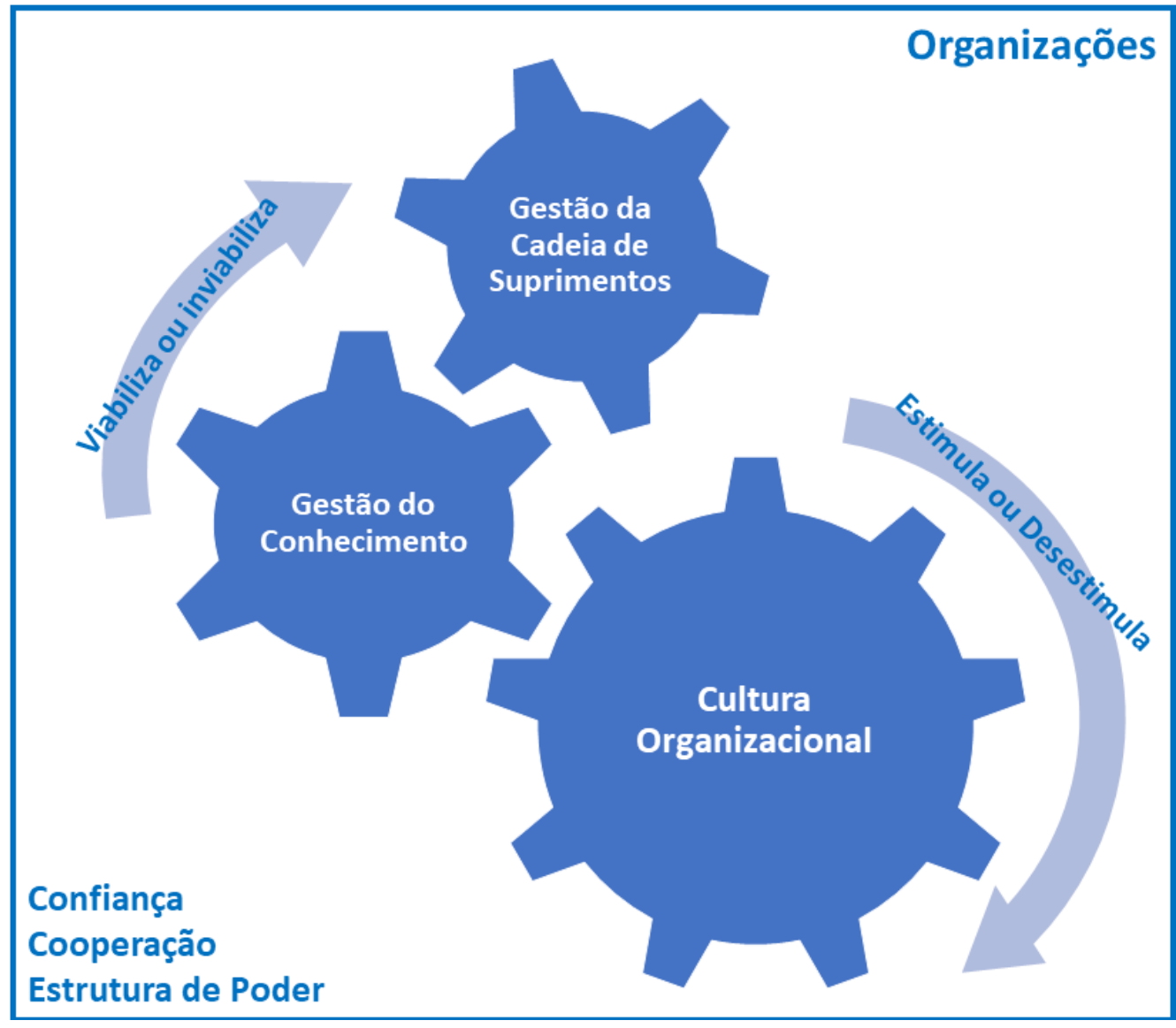

Fonte: Elaboração própria (2020)

A Figura 2 foi elaborada com base nos conceitos teóricos presentes na literatura, visando demonstrar essas interações. A cultura organizacional é a base para a GC e a GCS, pois sem uma cultura organizacional positiva em relação a construção, socialização e compartilhamento de dados, informação e conhecimento, dificilmente é possível implementar a GC em nível organizacional e, posteriormente, entre as organizações que compõem a mesma cadeia no âmbito da GCS.

Nesse sentido, a cultura deve dar suporte à implantação da GC, viabilizando por meio da confiança, cooperação e estrutura de poder a GCS e as demais estratégias que possuem a informação como recurso, propiciando que 
as organizações desempenhem melhor as suas atividades.

Cavalcante (2010, p. 24) reforça esse pensamento, ao evidenciar que a organização deve possuir ou desenvolver uma cultura positiva para o "[...] compartilhamento da informação e do conhecimento pelos atores organizacionais em todos os níveis organizacionais". Com isso, a cultura passa ser componente estratégico para as organizações que pretendem fazer uso da informação como componente estratégico em suas atividades.

Para Davenport e Prusak (1998) existem muitos tipos de cultura que podem impedir ou mesmo interromper a conversão de conhecimento. Assim, a cultura organizacional estimula ou desestimula a GC, ou, em outras palavras, a cultura organizacional pode promover ou impedir significativamente 0 sucesso da GC (TSENG, 2010). Nesse contexto, como a GCS trata da integração holística dos processos de negócios por intermédio da cadeia produtiva, a adequada implementação da GC viabiliza ou inviabiliza seu sucesso.

\section{CONSIDERAÇÕES FINAIS}

A relação entre a CO, a GC e GCS é real e direta, uma vez que a cultura contribui para efetividade dos processos de GC e GCS. Mais especificamente, pode-se destacar que essa forte relação se deve ao fato de que a cultura organizacional pode ser determinante para o estímulo ou desencorajamento do compartilhamento de conhecimento.

Em tese, o estímulo à adoção de práticas de compartilhamento de conhecimento pode proporcionar vantagens competitivas para a cadeia, entretanto, na prática o compartilhamento é uma ação difícil de ser realizada, devido aos valores individuais e organizacionais que influem tanto no contexto interno da organização quanto no coletivo da cadeia de suprimentos.

A partir desta constatação, sugere-se que as organizações devam desenvolver uma cultura voltada à valorização da informação enquanto recurso estratégico, focando no melhor uso da GC, apoiando as atividades de compartilhamento de conhecimento. Dessa maneira, o conhecimento gerado no 
ambiente organizacional, precisa ser compartilhado não apenas internamente, mas principalmente necessita ser compartilhado em rede, isto é, a cultura deve valorizar o compartilhamento de informações e conhecimento entre as organizações que compõem a cadeia de suprimentos.

Portanto, a fim de melhorar o desempenho organizacional é essencial cultivar uma cultura que incentive e forneça oportunidades para comunicar ideias, conhecimentos e experiências.

Recomenda-se um estudo que contemple as três temáticas, visando a comprovação efetiva da relação entre elas, além do aprofundamento das temáticas voltadas à cultura informacional, possibilitando a construção de modelos, ferramentas e métodos.

\section{REFERÊNCIAS}

ABDESSADAK, Jihane; ACHELHI, Hicham; REKLAOUI, Kamal. Innovation: The linking \& the impact of the variables "Knowledge Management" and "Organizational Culture" on the company's performance. In: INTERNATIONAL COLLOQUIUM ON LOGISTICS AND SUPPLY CHAIN MANAGEMENT, Tânger, Marrocos, 2018. Proceedings [...]. Tânger: IEEE, 2018. p. 170-174, Disponível em: https://ieeexplore.ieee.org/document/8428290. Acesso em: 22 nov. 2021.

ALMEIDA JÚNIOR, Oswaldo Francisco de. Mediação da informação: ampliando o conceito de disseminação. In: VALENTIM, Marta Ligia Pomim (org.). Gestão da informação e do conhecimento no âmbito da Ciência da Informação. São Paulo: Polis; Cultura Acadêmica, 2008. p. 41-54.

AL-TIT, Ahmad Adnan. Factors affecting the organizational performance of manufacturing firms. International Journal of Engineering Business Management. [S. I.], v. 9, p. 1-9, 2017. Disponível em: https://journalssagepubcom.ez87.periodicos.capes.gov.br/doi/pdf/10.1177/1847979017712628. Acesso em: 22 out. 2021.

BANDEIRA, Renata Albergaria de Mello; MELLO, Luiz Carlos Brasil de Brito; MAÇADA, Antonio Carlos Gastaud. Relacionamento interorganizacional na cadeia de suprimentos: um estudo de caso na indústria da construção civil. Produção, São Paulo, v. 19, n. 2, p. 376-387, maio/ago. 2009. Disponível em: https://www.redalyc.org/pdf/3967/396742036012.pdf. Acesso em: 14 ago. 2021.

CAVALCANTE, Luciane de Fátima Beckman. Gestão do comportamento informacional apoiada na cultura organizacional e em modelos mentais. 
Orientadora: Marta Ligia Pomim Valentim. 2010. 240 f. Dissertação (Mestrado em Ciência da Informação) - Programa de Pós-graduação em Ciência da Informação, Universidade Estadual Paulista, Marília, 2010.

$\mathrm{CHOO}$, Chun Wei. A organização do conhecimento: como as organizações usam a informação para criar significado, construir conhecimento e tomar decisões. São Paulo: SENAC Editora, 2003.

COUNCIL OF SUPPLY CHAIN MANAGEMENT PROFESSIONALS (CSCMP). Disponível em: https://cscmp.org/. Acesso em: 14 set. 2021.

DAVENPORT, Thomas H.; PRUSAK, Laurence. Conhecimento empresarial: como as organizações gerenciam o seu capital intelectual. 4. ed. Rio de Janeiro: Campus, 1998.

DIAS, Reinaldo. Cultura organizacional: construção, mudança e mudança. São Paulo: Atlas, 2013.

DYER, Jeffrey H.; SINGH, Harbir. The relational view: Cooperative strategy and sources of interorganizational competitive advantage. Academy of

Management Review, [S. I.], v. 23, n. 4, p. 660-679, oct. 1998. Disponível em: https://doi.org/10.5465/amr.1998.1255632. Acesso em: 14 dez. 2020.

FLEURY, Maria Tereza Leme; SHINYASHIKI, Gilberto; STEVANATO, Luiz Arnaldo. Arqueologia teórica e dilemas metodológicos dos estudos sobre cultura organizacional. In: MOTTA, Fernando Claudio Peres; CALDAS, Miguel P. (org.). Cultura organizacional e cultura brasileira. São Paulo: Atlas, 1997. p. 79-89.

GANESAN, Shankar. Determinants of long-term orientation in buyer-seller relationships. Journal of Marketing, [S. I.], v. 58, n. 2, p. 1-19, apr. 1994. Disponível em: https://doi.org/10.2307/1252265. Acesso em: 14 dez. 2021.

GIL, Antonio Carlos. Métodos e técnicas da pesquisa social. 6. ed. São Paulo: Atlas, 2008.

KAUFMANN, Lutz; CARTER, Craig R. International supply chain relationships and non financial performance: A comparison of US and German practices. Journal of Operations Management, [S. I.], v. 24, n. 5, p. 653-675, sep. 2006. Disponível em: https://doi.org/10.1016/j.jom.2005.07.001. Acesso em: 14 dez. 2021.

LAMBERT, Douglas M.; COOPER, Martha C.; PAGH, Janus D. Supply chain management: implementation issues and research opportunities. International Journal of Logistics Management, [S. I.], v. 9, n. 2, p. 1-20, 1998. Disponível em: https://doi.org/10.1108/09574099810805807. Acesso em: 14 set. 2021.

LI, Suhong; RAO, Subba S.; RAGU-NATHAN, T. S.; RAGU-NATHAN, Bhanu. Development and validation of a measurement instrument for studying supply chain management practices. Journal of Operations Management, [S. I.], v. 
23, n. 6, p. 618-641, sep. 2005. Disponível em:

https://doi.org/10.1016/j.jom.2005.01.002. Acesso em: 14 set. 2021.

LUMMUS, Rhonda R.; VOKURKA, Robert J. Defining supply chain management: A historical perspective and practical guidelines. Industrial Management Data Systems, [S. I.], v. 99, n. 1, p. 11-17, 1999. Disponível em: https://doi.org/10.1108/02635579910243851. Acesso em: 14 ago. 2021.

MACHLINE, Claude. Cinco décadas de logística empresarial e administração da cadeia de suprimentos no Brasil. RAE, São Paulo, v. 51, n. 3, p. 227-231, maio/jun. 2011. Disponível em: https://doi.org/10.1590/S003475902011000300003. Acesso em: 14 dez. 2021.

MARCONI, Marina de Andrade; LAKATOS, Eva Maria. Técnicas de pesquisa: planejamento e execução de pesquisas, amostragens e técnicas de pesquisa, elaboração, análise e interpretação dos dados. São Paulo: Atlas, 2006.

MELNYK, Steven A.; LUMMUS, Rhonda; VOKURKA, Robert J.; SANDOR, Joseph. Supply chain management 2010 and beyond: Mapping the future of strategic supply chain. East Lansing, MI: APICS Educational and Research Foundation, 2007. Disponível em:

http://www.apics.org/docs/about/scm_report_web_sm.pdf. Acesso em: 14 dez. 2021.

MENTZER, John T.; DEWITT, William; KEEBLER, James S.; MIN, Soonhong; NIX, Nancy W.; SMITH, Carlo D.; ZACHARIA, Zach G. Defining supply chain management. Journal of Business Logistics, [S. I.], v. 22, n. 2, 2001.

Disponível em: https://onlinelibrary-

wiley.ez87.periodicos.capes.gov.br/doi/abs/10.1002/j.2158-

1592.2001.tb00001.x. Acesso em: 04 dez. 2021.

MIN, Soonhong; MENTZER, John T. Developing and measuring supply chain management concepts. Journal of Business Logistics, [S. I.], v. 25, n. 1, p. 63-99, 2004. Disponível em: https://doi.org/10.1002/j.21581592.2004.tb00170.x. Acesso em: 14 dez. 2021.

MUNSON, Charles L.; ROSENBLATT, Meir; ROSENBLATT, Zehava. The use and abuse of power in supply chains. Business Horizons, [S. I.], v. 42, n. 1, p. 55-65, 1999. Disponível em: https://www.sciencedirect.com/science/article/abs/pii/S0007681399800494. Acesso em: 4 nov. 2021.

NIELSEN, Bo Bernhard. The role of trust in collaborative relationships: a multidimensional approach. M@n@gement, [S. I.], v. 7, n. 3, p. 239-256, 2004. Disponível em: https://www.cairn.info/revue-management-2004-3-page239.htm. Acesso em: 18 dez. 2020.

NONAKA, Ikujiro; TAKEUCHI, Hirotaka. Criação de conhecimento na empresa. Rio de Janeiro: Campus, 1997. 
OLIVEIRA, Dayane Mayely Silva; COHEN Max Fortunato. Os usos da TI ao longo da cadeia de suprimentos e em conjunto com as principais técnicas colaborativas e de gestão. Revista Eletrônica de Sistemas de Informação, [S. I.], v. 9, n. 2, p. 1-22, 2010. Disponível em:

https://search.proquest.com/docview/860073320?pq-origsite=gscholar. Acesso em: 18 dez. 2021.

PARRA, Paulo H.; PIRES, Sílvio R. I. Análise da gestão da cadeia de suprimentos na indústria de computadores. Revista Gestão e Produção, São Carlos, v. 10, n. 1, p. 1-15, 2003. Disponível em: http://dx.doi.org/10.1590/S0104-530X2003000100002. Acesso em: 18 dez. 2021.

PIRES, Sílvio R. I. Gestão da cadeia de suprimentos (supply chain management): conceitos, estratégias, práticas e casos. 2. ed. São Paulo: Atlas, 2009.

PIRES, Sílvio R. I.; BREMER, Carlos F.; SANTA EULÁLIA, Luis A. de; GOULART, Christiane P. Supply chain and virtual enterprises: Comparisons, migration and a case study. International Journal of Logistics: Research and Applications, [S. I.], v. 4, n. 3, p. 297-311, 2001.

PONJUÁN DANTE, Gloria. Gestión de información: dimensiones e implementación para el éxito organizacional. Rosario: Nuevo Paradigma, 2004.

RITTER, Michael. Cultura organizacional. Buenos Aires: La Crujía, 2008.

ROBBINS, Stephen P. Comportamento organizacional. 11. ed. São Paulo: Pearson Prentice Hall, 2007.

SCHEIN, E. Organizational culture and leadership. San Francisco: Bess, 1995.

SUTTON, Michael J. D. Examination of the historical sensemaking processes representing the development of knowledge management programs in universities. 2007. 441 p. Thesis (Doctor of Philosophy) Faculty of Education, McGill University, Montreal, 2007. Disponível em: https://issuu.com/academic-conferences.org/docs/sutton_km_dissertation. Acesso em: 18 nov. 2021.

TAKEUCHI, Hirotaka; NONAKA, Ikujiro. Gestão do conhecimento. Porto Alegre: Bookman, 2008.

TAN, Keah Choon. A framework of supply chain management literature. European Journal of Purchasing \& Supply Management, [S. I.], v. 7, n. 1, p. 39-48, mar. 2001. Disponível em: https://doi.org/10.1016/S09697012(00)00020-4. Acesso em: 18 dez. 2021.

TSENG, Shu-Mei. The correlation between organizational culture and knowledge conversion on corporate performance. Journal of Knowledge 
Management, [S. I.], v. 14, n. 2, p. 269-284, 2010. Disponível em: https://wwwemerald.ez87.periodicos.capes.gov.br/insight/content/doi/10.1108/1367327101 1032409/full/html. Acesso em: 18 dez. 2021.

VALENTIM, Marta Ligia Pomim. Inteligência competitiva em organizações: dado, informação e conhecimento. DataGramaZero, Rio de Janeiro, v. 3, n. 4, p. 1-13, 2002.

VALENTIM, Marta Ligia Pomim. Gestão da informação e gestão do conhecimento: especificidades e convergências. Londrina: Infohome, 2004. Disponível em: http://www.ofaj.com.br/colunas_conteudo.php?cod=88. Acesso em: 18 dez. 2021.

VALENTIM, Marta Ligia Pomim. Gestão da informação e gestão do conhecimento em ambientes organizacionais: conceitos e compreensões.

Tendências da Pesquisa Brasileira em Ciência da Informação, v. 1, n. 1, 2008. Disponível em: http://hdl.handle.net/20.500.11959/brapci/119521. Acesso em: 18 dez. 2021.

VALENTIM, Marta Ligia Pomim; JORGE, Carlos Francisco Bitencourt; CERETTA-SORIA, María Gladys. Contribuição da competência em informação para os processos de gestão da informação e do conhecimento. Em Questão, Porto Alegre, v. 20, n. 2, p. 207-231, 2014. Disponível em:

https://seer.ufrgs.br/EmQuestao/article/view/48642/32122. Acesso em: $18 \mathrm{dez}$. 2021.

VIVALDINI, Mauro; PIRES, Sílvio R. I. Operadores logísticos: integrando operações em cadeias de suprimento. São Paulo: Atlas, 2010.

WEN, Hua; GU, Qianling. The elements of supply chain management in new environmental era. In: INTERNATIONAL CONFERENCE ON MANAGEMENT SCIENCE AND ENGINEERING MANAGEMENT, 7., Berlim, 2014.

Proceedings [...] Berlim: Springer, 2017. p. 867-880. Disponível em: https://link.springer.com/chapter/10.1007/978-3-642-40081-0_74. Acesso em: 18 dez. 2021.

WIIG, K. Knowledge sense of the organization: Thinking about thinking. How people and organizations create, represent and use knowledge. Arlington, TX: Schema Press, 1993.

\title{
CONTRIBUTION OF ORGANIZATIONAL CULTURE TO KNOWLEDGE MANAGEMENT IN THE CONTEXT OF SUPPLY CHAIN MANAGEMENT
}

\author{
ABSTRACT \\ Objective: It was sought to better understand the object researched, from the approach
}


of supply chain management, debating in an interrelated way the contributions of organizational culture and knowledge management. Methodology: This is a research an approach qualitative and bibliographic type. Results: It was found that the relationship between organizational culture, knowledge management and supply chain management is real and direct since culture contributes to the effectiveness of the processes of both management models. It is noteworthy that the strong relationship is due to the fact that the organizational culture is decisive for stimulating or discouraging knowledge sharing. In theory, stimulating the adoption of knowledge sharing practices can provide competitive advantages for the chain, however, in practice sharing is a difficult action to be performed, due to the individual and organizational values that influence both in the internal context of the organization and in the collective of the supply chain. Conclusions: Based on this observation, it is suggested that organizations should develop a culture aimed at valuing information as a strategic resource, focusing on the best use of knowledge management, supporting sharing activities. In order to improve organizational performance, it is essential to cultivate a culture that encourages and provides opportunities to communicate ideas, knowledge, and experiences.

Descriptors: Organizational Culture. Knowledge Management. Supply Chain Management. Knowledge Sharing.

\title{
CONTRIBUCIÓN DE LA CULTURA ORGANIZACIONAL A LA GESTIÓN DEL CONOCIMIENTO EN EL CONTEXTO DE LA GESTIÓN DE LA CADENA DE SUMINISTRO
}

\begin{abstract}
RESUMEN
Objetivo: Se buscó comprender mejor el objeto investigado, desde el enfoque de la gestión de la cadena de suministro, debatiendo de manera interrelacionada los aportes de la cultura organizacional y la gestión del conocimiento. Metodología: Es una investigación cualitativa, de tipo bibliográfico. Resultados: Se encontró que la relación entre cultura organizacional, gestión del conocimiento y gestión de la cadena de suministro es real y directa, ya que la cultura contribuye a la efectividad de los procesos de ambos modelos de gestión. Es de destacar que la fuerte relación se debe a que la cultura organizacional es determinante para estimular o desalentar el intercambio de conocimientos. En teoría, estimular la adopción de prácticas de intercambio de conocimiento puede brindar ventajas competitivas para la cadena, sin embargo, en la práctica compartir es una acción difícil de realizar, debido a los valores individuales y organizacionales que influyen, tanto en el contexto interno de la cadena. organización y en el colectivo de la cadena de suministro. Conclusiones: A partir de esta observación, se sugiere que las organizaciones desarrollen una cultura orientada a valorar la información como recurso estratégico, enfocándose en el mejor uso de la gestión del conocimiento, apoyando las actividades de intercambio. Para mejorar el desempeño organizacional, es fundamental cultivar una cultura que fomente y brinde oportunidades para comunicar ideas, conocimientos y experiencias.
\end{abstract}

Descriptores: Cultura Organizacional. Gestión del Conocimiento. Gestión de la Cadena de Suministro. Compartir Conocimiento. 
Ana Livia Cazane, Marta Lígia Pomim Valentim

Contribuição da cultura organizacional para a gestão do conhecimento no contexto da gestão da cadeia de suprimentos

Recebido em: 24.04.2021

Aceito em: 22.12.2021 